\title{
Breast Cancer in the Elderly: An Observational Study Investigating Compliance of Screening Mammography in an Underserved Community
}

\author{
Shruti Sharma ${ }^{\mathrm{a}, \mathrm{c}} \mathbb{C}^{\circ}$, Dixita Patel ${ }^{\mathrm{a}}$, Sushma Pavuluria ${ }^{\mathrm{a}}$, Amy Stein ${ }^{\mathrm{b}}$, Binal Patel ${ }^{\mathrm{a}}$, \\ Nadia Qureshi ${ }^{\mathrm{a}}$, Imran Hasnuddin ${ }^{\mathrm{a}}$, Tsvetelina Todorova ${ }^{\mathrm{a}}$, \\ Krishnan Srinivasan ${ }^{\mathrm{a}}$, Masood Ghouse ${ }^{\mathrm{a}}$
}

\begin{abstract}
Background: The incidence of breast cancer increases with age. Individuals living in higher socioeconomic communities also have higher incidence secondary to early detection of breast cancer from increased accessibility to mammograms. This retrospective study studied the percentage of new breast cancer cases in the elderly between 2010 and 2019 , and investigated the compliance of screening mammography in some of the medically underserved suburbs of southern Chicago.
\end{abstract}

Methods: The parameters used to power this study include "age greater than 70" and "2010 to present" at the time the study was first initiated. The final data set contained 381 electronic health records (EMRs) that met the parameters of interest. We specifically looked at method of diagnosis, stage at diagnosis, date of last normal screening mammogram, hormone status, histology, race, and smoking history.

Results: Thirty percent of the breast cancer patients diagnosed at our institution were over 70 years of age between 2010 and 2019. Of the 381 patients included in the overall sample, $45 \%$ were diagnosed with breast cancer by screening mammogram, and $52 \%$ of individuals in the $70-75$ age group were diagnosed with breast cancer by screening mammography. Only $40 \%$ of individuals in the $75+$ age group were diagnosed with breast cancer by screening mammogram $(\mathrm{P}=0.0234)$. Furthermore, in the overall sample, $63 \%$ had a normal screening mammogram at some time prior to their breast cancer diagnosis. In the 70 - 75 age group, $76 \%$ had a normal screening mammogram at some time prior to their breast cancer diagnosis. In the 75+ age group, only $54 \%$ had a normal screening mammogram at some time prior to their breast cancer diagnosis $(\mathrm{P}<0.0001)$. Individuals in both age

Manuscript submitted August 26, 2021, accepted September 30, 2021

Published online October 21, 2021

${ }^{a}$ Comprehensive Cancer Institute, Franciscan Health Olympia Fields, 3900 West 203rd Street, Olympia Fields, IL 60461, USA

bOffice of Research and Sponsored Programs, Midwestern University, 19555 N 59th Avenue, Glendale, AZ 85308, USA

${ }^{\mathrm{c} C o r r e s p o n d i n g ~ A u t h o r: ~ S h r u t i ~ S h a r m a, ~ C o m p r e h e n s i v e ~ C a n c e r ~ I n s t i t u t e, ~}$ Franciscan Health Olympia Fields, 3900 West 203rd Street, Olympia Fields, IL 60461, USA. Email: Shruti.Sharma@franciscanalliance.org

doi: https://doi.org/10.14740/wjon1397 groups were more likely to have early-stage breast cancers and luminal A hormone expression.

Conclusions: Decreased compliance to screening mammography is observed in the elderly living in underserved communities. Since the elderly are underrepresented in research, organizations do not have sufficient information to recommend screening mammography in the elderly. With increasing life expectancy, observational studies have demonstrated a mortality benefit with screening mammography by early detection of breast cancer, favorable breast cancer characteristics and potentially higher cure rates. Socioeconomic factors also affect screening compliance and likely influenced the results of our study. Future studies should investigate how individual factors influence screening mammography compliance in the elderly in underserved communities.

Keywords: Breast cancer; Prevention; Diagnosis; Cancer epidemiology; Underserved communities; Elderly

\section{Introduction}

Breast cancer is the most common malignancy diagnosed among women in the world, accounting for $11.7 \%$ of all new cancer cases [1]. Some risk factors for developing breast cancer later in life include age, postmenopausal hormone use, age of first birth, obesity, alcohol, smoking, and gene mutations [2, 3]. Incidence rates appear to increase with age and are higher in economically developed areas secondary to increased breast cancer screening [1]. Screening mammograms facilitate early detection and treatment of breast cancer, reducing the odds of mortality from breast cancer itself. Although most organizations recommend starting mammogram screening at the age of 50, there is no clear census regarding the age to stop screening (Table 1).

With increasing life expectancy, the incidence of breast cancer in individuals of 75+ years is 400 per 100,000 individuals according to Surveillance, Epidemiology and End Results Program [4]. There are no guidelines regarding screening mammograms for the "elderly". Although the "elderly" is often defined as having a chronological age of $65+$ years, there is no biological evidence to support this definition. Furthermore, 
Table 1. A Comparison of National Breast Cancer Guideline Screening

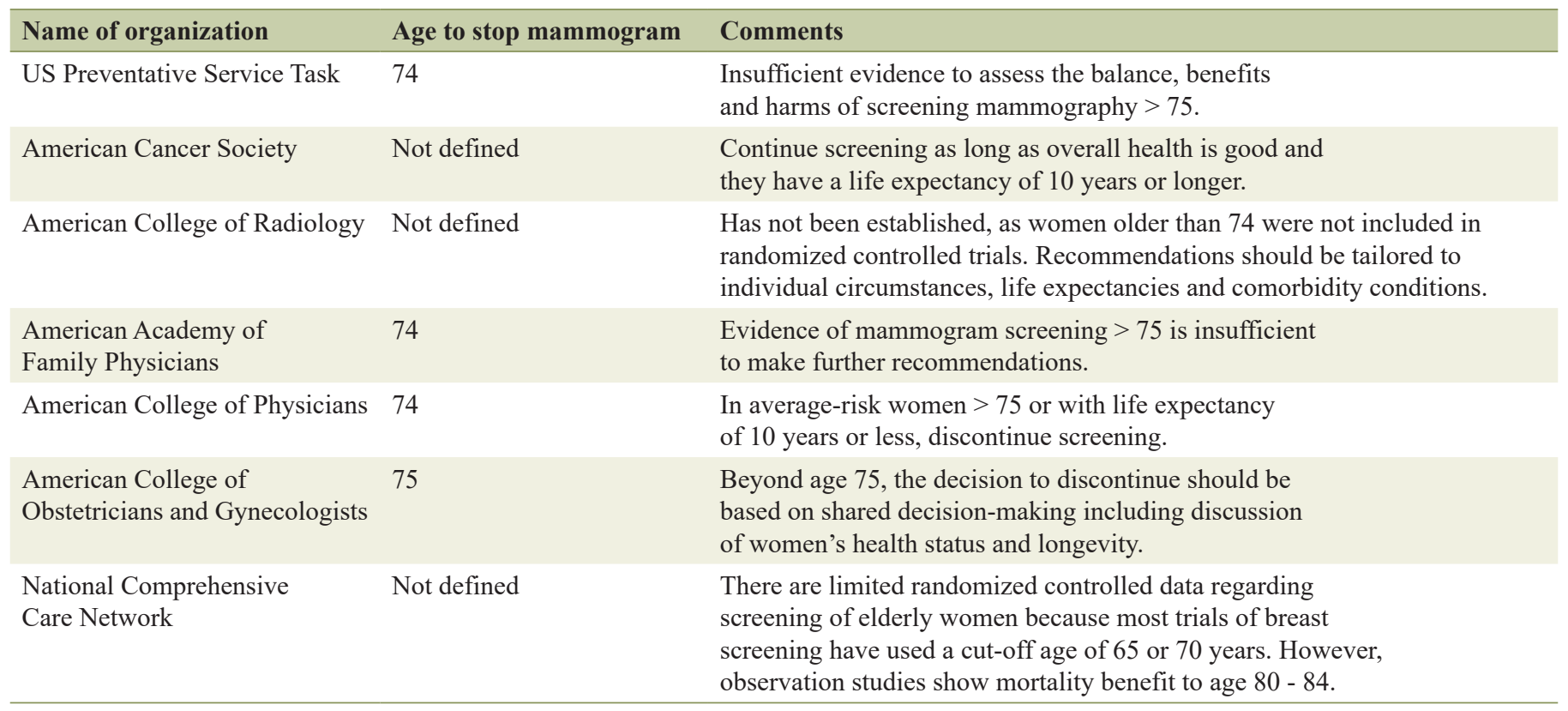

breast cancer guidelines also appear to have inconsistent recommendations for breast cancer screening in individuals greater than 70 years of age. The benefits and harms of screening in the elderly population are also less clear since randomized controlled trials of screening rarely include this age group [5]. Often the harms of screening mammograms are amplified in older adults. Their lack of education and cognitive decline in the elderly is thought to interfere with their ability to truly give informed consent to screening mammograms [5].

Since the incidence of breast cancer is influenced by age and socioeconomic factors, this retrospective observation study intends to investigate the percentage of new breast cancer cases in the elderly in a given period of time, and determine the compliance of screening mammography at our institution to further advocate for breast cancer prevention in the elderly living in underserved areas. Our institution provides several healthcare services for patients living in surrounding medically underserved areas such as Chicago Heights and Ford Heights in southern Chicago. To our knowledge, this is the first study to investigate breast cancer screening compliance in individuals older than 70 years of age that are also living in underserved areas.

\section{Materials and Methods}

To compare our institution to facilities across all states, we used the National Cancer Database (NCDB) to estimate the percentage of new breast cancer cases at our facility at present. We looked at the NCBD Public Benchmark Report Breast Cancer Cases diagnosed between 2009 and 2018, since this is what is publicly available at this time $[6,7]$. The cases included were those that were diagnosed at our institution but received all/part of their first course of treatment elsewhere (class of case 00), and those that were diagnosed at our institu- tion and received all/part of their first course of treatment at the reporting cancer institution (class of case 1 - 14). We then queried based on age group, the hospital type, the type of cases we were looking for, and geography $[6,7]$.

We then extracted data in the last 10 years between 2010 and 2019, from our institution's breast cancer tumor registry. The parameters used to power the study included "age greater than 70" and "2010 to present". Initially, 402 records met these parameters, however, duplicate records and invalid medical records were removed. The final data set contained 381 electronic health records (EMRs) that met the parameters of interest.

The following data were extracted from our tumor registry: date, method, stage, age of breast cancer diagnosis, last normal screening mammogram prior to diagnosis, hormone status, histology, race, and smoking history. The method of diagnosis was described as either screening mammogram, diagnostic mammogram or other (CT imaging studies). Staging included three categories: stage 0 (ductal carcinoma in situ (DCIS) and lobular carcinoma in situ (LCIS)), early stage (stage I and stage II), and advanced stage (stage III and stage IV). The age of diagnosis was divided between the categories of 70 - 75 years and 75+ years. Hormone status included evaluation of estrogen, progesterone and human epidermal growth factor 2 (HER-2) status and was described as either positive, negative, or equivocal. Histology was simplified to include DCIS and LCIS, invasive ductal and lobular carcinoma, and mixed histology. The race of the individual included Caucasian, African American or other. Smoking history was described as: current, former, or never.

\section{Ethics}

Since our data did not involve experimentation with human subjects and was retrospective in nature, our institutional re- 


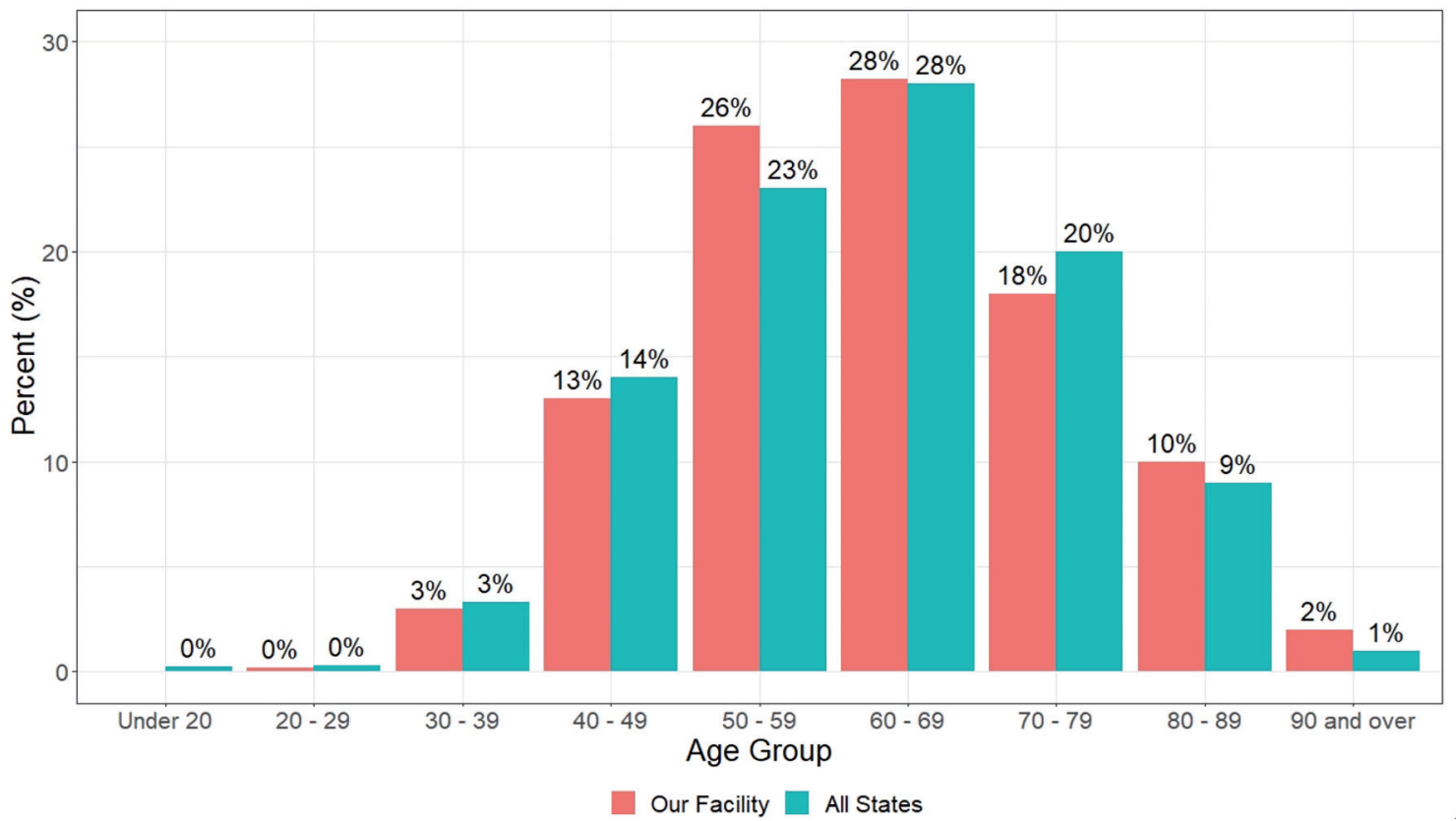

Figure 1. The percentage of new breast cancer cases in the elderly at our institution compared to all states between 2009 and 2018.

view board waived informed consent for our study. This study was conducted in compliance with the ethical standards of the responsible institution on human subjects as well as the Helsinki Declaration.

\section{Statistical analysis}

All categorical variables were summarized with percentage and (count). Comparisons between and within age groups across different variables were analyzed with a Chi-square test or Fisher's exact test for smaller sample sizes. The time from last normal screening mammogram is approximate and was calculated by subtracting the year of the last normal screening mammogram and the year of diagnosis. Comparison between groups of the time from last normal screening was analyzed using a Mann-Whitney U test. Statistical significance was assessed at the 0.05 level and analysis was done using $\mathrm{R}$ version 3.6.0.

\section{Results}

\section{New cases of breast cancer}

Compared to all the states, the percentage of new breast cancer cases in individuals greater than 70 years of age is reported to be $30.8 \%(n=300,317$, total $=972,733, P=0.3084$, Fig. 1) $[6,7]$ between 2009 and 2018 per the National Cancer Database. In our institution, there were 1,262 total breast cancer cases reported between 2010 and 2019, including both male and female cases [8]. At our institution, the percentage of new breast cancer cases in individuals greater than 70 years of age is reported to be $30.2 \%(\mathrm{n}=381$, total $=1,262)$.

\section{Summary of demographic variables}

The summary of all variables overall and by age group can be found in Table 2. We specifically looked at three comparing groups in this study: the overall sample $(n=381)$, the $70-75$ age group, and 75+ age group.

\section{Screening mammography}

Of the 381 patients included in the study, 45\% (171) were diagnosed with breast cancer by screening mammogram. Significantly, more patients in the 70 - 75 years group, were diagnosed with breast cancer by screening mammogram, 52\% (85) compared to $40 \%(86)$ in the $75+$ age group $(\mathrm{P}=0.0234)$.

\section{Smoking}

For smoking status in the overall sample, 5\% (19) were current smokers, 38\% (142) were former smokers, and 56\% (209) never smoked. Significantly, there were more former smokers in the 70 - 75 age group, $44 \%$ (71) vs. $34 \%$ (71) in the $75+$ age group $(\mathrm{P}=$ $0.0497)$. There were also significantly less never smokers in the 70 - 75 age group $49 \%$ (79) compared to $62 \%$ (130) in the $75+$ age group ( $\mathrm{P}=0.0213)$. There was no difference in current smokers between the $70-75$ age group and $75+$ age group $(P=0.5416)$.

\section{Hormone status}

For hormone status in the overall sample, $81 \%$ (294) of the breast cancer tumors expressed estrogen, 77\% (272) proges- 
Table 2. Summary of Demographic Variables Using \% (Count)

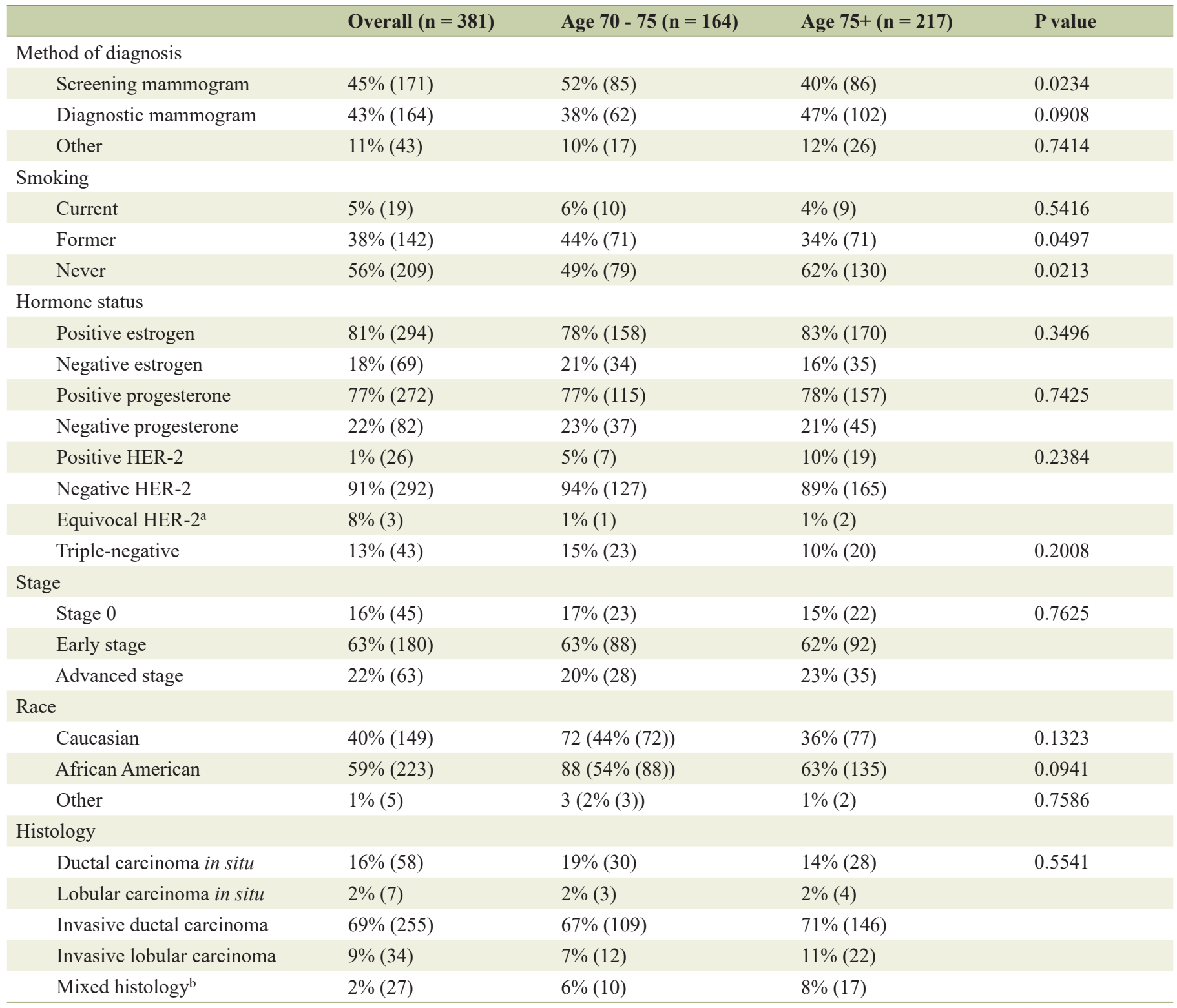

aNo further workup of clarification of equivocal status was found in chart review. bMixed histology includes overlapping histology of the above and new histology patterns (marginal zone B-cell lymphoma, spindle cell carcinoma, undifferentiated, unknown). HER-2: human epidermal growth factor 2.

terone, and 1\% (26) expressed HER-2. Furthermore, $18 \%$ (69) were estrogen negative, 22\% (82) progesterone negative, and 91\% (292) HER-2 negative. Approximately 8\% (3) had equivocal HER-2 status and no further workup including fluorescence in situ hybridization (FISH) was identified to clarify HER-2 status. Lastly, 13\% (43) were classified as triple-negative. There were no differences in hormone status in the $70-75$ age group or $75+$ age group (all $\mathrm{P}>0.05$ ).

\section{Staging}

In the overall sample, $16 \%$ (45) of the individuals were diag- nosed with stage $0,63 \%$ (180) were diagnosed with early stage breast cancer and $22 \%$ (63) were diagnosed with advanced stage breast cancer. There were no differences in cancer stage in the $70-75$ age group, or $75+$ age group $(\mathrm{P}=0.7625)$.

\section{Histology}

In the overall sample, there were $16 \%$ (58) DCIS, $2 \%$ (7) LCIS, $69 \%$ (255) invasive ductal carcinoma, 9\% (34) invasive lobular carcinoma and 2\% (27) mixed histology patterns identified. Mixed histology included overlapping histology of the above and new histology patterns. Patterns that were observed in our 


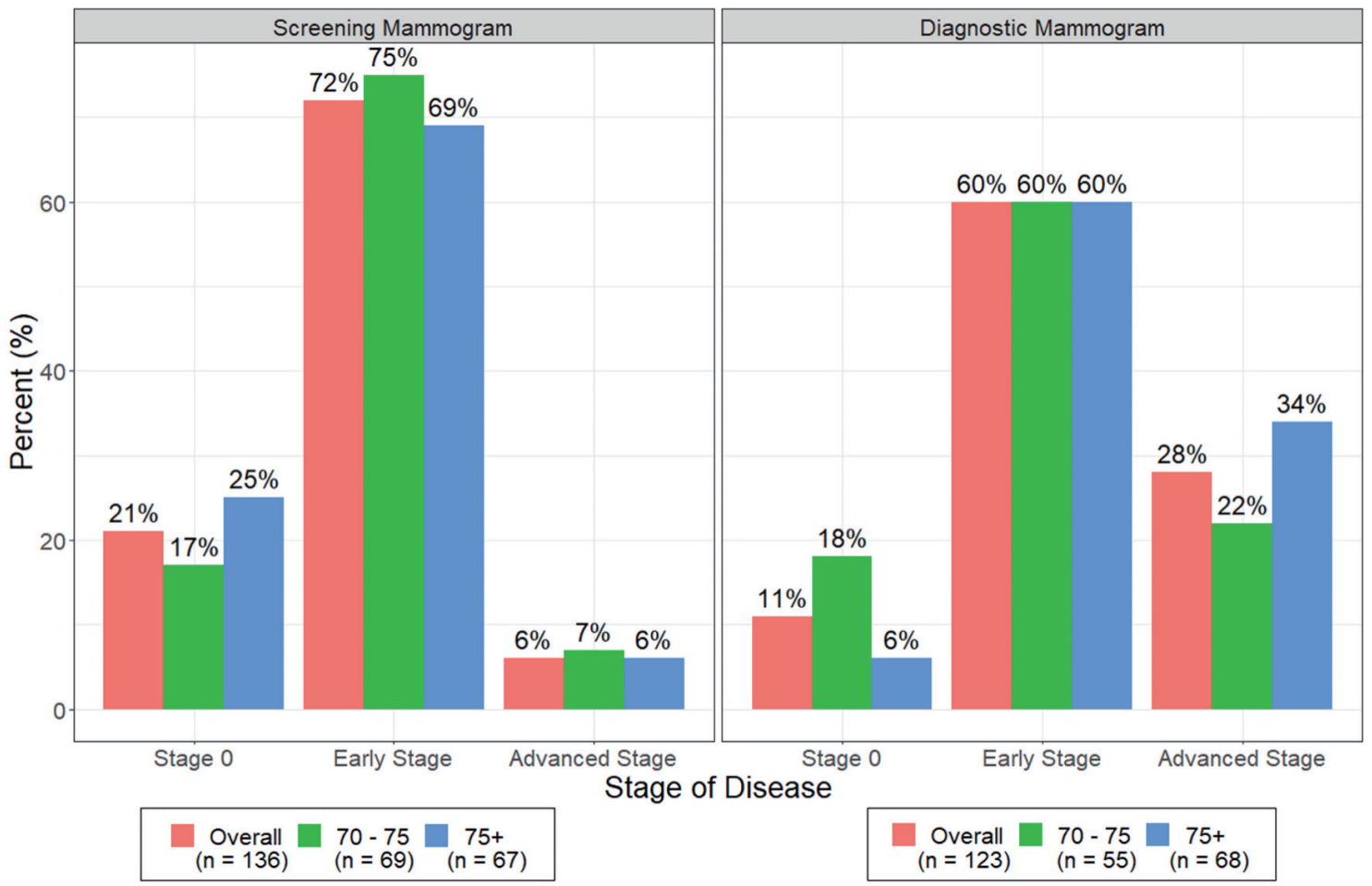

Figure 2. Relationship between method of diagnosis, stage of cancer at diagnosis, and age group.

sample included marginal zone B-cell lymphoma, spindle cell carcinoma, undifferentiated carcinomas, and unknown. There were no differences in histology in the $70-75$ age group or $75+$ age group $(\mathrm{P}=0.5541)$.

\section{Screening mammography prior to breast cancer diagnosis}

In the overall sample, $63 \%$ (234) had a normal screening mammogram at some time prior to their breast cancer diagnosis. The median length of time from the last normal screening mammogram to the time of diagnosis was approximately 2 years but ranged from 0 to 19 years. In the 70 - 75 age group, $76 \%$ (119) had a normal screening mammogram prior to their diagnosis of breast cancer compared to only $54 \%$ (115) in the $75+$ years age group $(\mathrm{P}<0.0001)$. The length of time from the last normal screening mammogram to the time of their diagnosis of breast cancer in the 70 - 75 age group was significantly shorter than in the $75+$ age group, a median of approximately 1 year vs. 2 years $(\mathrm{P}=0.0087)$.

\section{Screening mammography and stage of breast cancer at di- agnosis}

In the overall sample, approximately 136 individuals obtained a screening mammogram and had available data on cancer stage. Of these individuals, $21 \%$ were diagnosed with stage 0 breast cancer, $72 \%$ with early stage breast cancer and $6 \%$ with advanced stage cancer. In the $70-75$ age group, approximately
69 individuals obtained a screening mammogram and had data available on cancer staging. Of these individuals, $17 \%$ were diagnosed with stage 0 breast cancer, $75 \%$ with early stage breast cancer and $7 \%$ with advanced breast cancer. In the $75+$ age group, approximately 67 individuals obtained a screening mammogram and had available data on cancer stage. Of these individuals, $25 \%$ were diagnosed with stage 0 breast cancer, $69 \%$ with early stage breast cancer and $6 \%$ with advanced stage breast cancer (Fig. 2).

\section{Screening mammography and hormone expression at di- agnosis}

Of the 171 individuals that obtained a screening mammogram in the overall sample, 3\% expressed triple-positive hormone status, $6 \%$ expressed triple-negative hormone status, and $63 \%$ expressed luminal A. Of the 85 individuals that obtained a screening mammogram in the $70-75$ age group, $1 \%$ expressed triple-positive hormone status, 7\% expressed triple-negative hormone status and $65 \%$ expressed luminal A hormone status. Of the 86 individuals that obtained a screening mammogram in the $75+$ age group, $5 \%$ expressed triple-positive hormone status, $12 \%$ expressed triple-negative hormone status and $60 \%$ expressed luminal A hormone status (Fig. 3).

\section{Race and hormone expression at diagnosis}

The relationship between race and hormone status of breast 


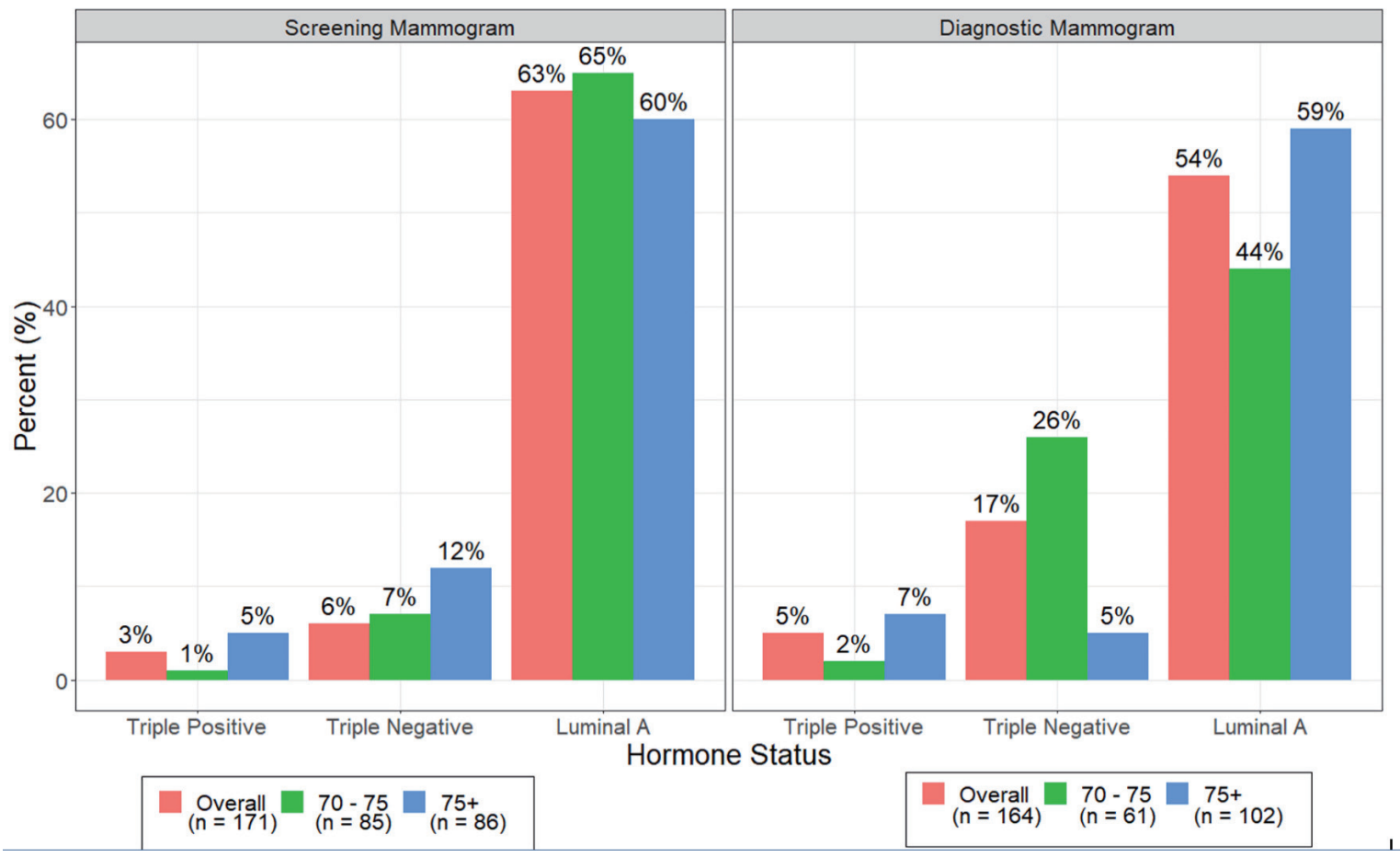

Figure 3. Relationship between method of diagnosis, hormone status of cancer at diagnosis, and age group.

cancer within the $70-75$ age group and $75+$ age group was considered (Table 3 ). There was no difference between races for triple-positive $(70-75: \mathrm{P}=0.5672,75+: \mathrm{P}=0.9303)$, triple-negative $(70-75: \mathrm{P}=0.7819,75+: \mathrm{P}=0.1139)$, or luminal A type hormone status $(70-75: \mathrm{P}=0.2100,75+: \mathrm{P}=0.4620)$.

\section{Discussion}

Between 1960 and 2015, the life expectancy for the total population in the USA has increased by almost 10 years from 67.7 to 79.4 years. Women, particularly, are projected in live to age 87.3 years by the year 2060 [9]. As life expectancy of women increases, the definition of elderly becomes difficult to define. As a direct result, it becomes important to reexamine breast cancer screening guidelines that have been created by major organizations. Although many organizations agree starting mammogram screening by age 50 , there is no clear census regarding the age to stop mammogram screening. These equivocal guidelines adversely affect screening mammography utility in the elderly population.

Breast cancer incidence in the USA is associated with socioeconomic status as measured at both the individual and community level $[2,10]$. One study examined this direct association and found that individual factor, such as higher education, was associated with a greater risk of developing breast cancer. Furthermore, living in higher economically developed areas was also associated with a greater risk of developing breast cancer. One possible explanation for this observation was that individuals had more accessibility to mammograms, leading to increased breast cancer incidence through early detection [10]. Since many of our patients are from lower socioeconomically developed areas, we considered that the actual percentage of new breast cancer cases at our institution may be higher than what was observed. In our study we found only $30 \%$ of our breast cancer patients were over 70 years of age between 2010 and 2019. This prompted us to evaluate compliance of screening mammography in the elderly.

Breast cancer incidence increases with age until the seventh decade and then is noted to decrease. Decreased incidence

Table 3. Relationship Between Hormone Status of Cancer and Race by Age Group

\begin{tabular}{|c|c|c|c|c|c|c|}
\hline & \multicolumn{3}{|c|}{ Ages $70-75(n=163)$} & \multicolumn{3}{|c|}{ Ages $75+(n=214)$} \\
\hline & $\begin{array}{l}\text { African American } \\
(\mathrm{n}=72)\end{array}$ & $\begin{array}{l}\text { Caucasian } \\
(\mathrm{n}=\mathbf{8 8})\end{array}$ & P value & $\begin{array}{l}\text { African American } \\
(\mathrm{n}=77)\end{array}$ & $\begin{array}{l}\text { Caucasian } \\
(\mathrm{n}=135)\end{array}$ & P value \\
\hline Triple-negative & $15 \%(11)$ & $13 \%(11)$ & 0.7819 & $14 \%(11)$ & $7 \%(9)$ & 0.1139 \\
\hline Luminal A & $51 \%(37)$ & $63 \%(55)$ & 0.2100 & $55 \%(42)$ & $61 \%(82)$ & 0.4620 \\
\hline
\end{tabular}

The $\mathrm{P}$ value is based on a Chi-square test comparing the proportion of race within each age group. 
rates observed in women $80+$ years may reflect lower rates of screening $[11,12]$. In our sample, it was noted that only $45 \%$ were diagnosed with breast cancer by screening mammogram. In the $75+$ age group, only $40 \%$ of individuals were diagnosed with breast cancer by screening mammogram. This forced us to investigate whether individuals had screening mammograms prior to their breast cancer diagnosis. We observed that more than $60 \%$ of the total sample and more than $50 \%$ in the $75+$ age group had normal screening mammograms at some time prior to their breast cancer diagnosis, similar to the data presented by the National Center of Health Sciences, who cited that $50.6 \%$ of individuals $75+$ years were observed to have a screening mammography within 2 years of their study [13]. Furthermore, elderly patients that were $<100 \%$ below poverty line, less educated and had difficulty learning were noted to have decreased screening mammography percentages compared to those individuals that were $400 \%+$ above poverty line and more educated [13]. These statistics suggest that elderly individuals living in underdeveloped communities may not be properly educated regarding the importance of screening mammography in breast cancer prevention. Importantly, one study cites that the primary reason women do not proceed with screening mammography is lack of physician recommendation [14]. This study examined screening mammography practices of middle age and older women and identified personal demographics, perceived health status and health care access factors that were associated with the time in which the participants $(\mathrm{n}=1,242)$ reported their most recent screening mammography [14]. In the $75+$ age group, only $12.7 \%$ of individuals had screening mammograms within 1 year and $17.3 \%$ of individuals had screening mammograms within 2 years, which was less than the younger age groups. Furthermore, individuals in the 75+ years had the least routine follow-up with medical providers in the last 12 months compared to the younger age groups [14]. Comparing this to our study, individuals of 75+ years also had longer lengths of time from their last normal screening mammogram to their breast cancer diagnosis by 1 year (median difference); however, the range was noted to be broad for both age groups. These studies suggest that the elderly living in underdeveloped areas may not have accessibility to health care leading to delay in breast cancer diagnosis and treatment. Other possible reasons for decreased screening in the elderly include overemphasis of comorbidities, concern for overdiagnosis and overtreatment of indolent disease and lack of standard evaluation by clinicians. It is important to objectively evaluate the benefits and harms of screening in the elderly.

At least 20 years ago, it was acknowledged by the scientific community that the number of elderly patients with breast cancer was increasing and the knowledge regarding differences in biology and clinical outcomes of breast cancer in the elderly population was limited [15]. It was also acknowledged that there was under enrollment of patients older than 65 years in clinical trials [15]. Although observational studies provide valuable information regarding screening in the elderly, they do not substitute for highly regulated clinical trials, making it difficult for organizations to compare observational studies and standardize recommendations regarding screening of malignancies in the elderly. As a direct consequence, it makes decision making for advocating screening mammograms in the elderly more challenging and subjective for clinicians, resulting in decreasing breast cancer screening.

Screening mammography decreases breast cancer mortality by detecting breast cancers of small size and early stage. Randomized control trials show at least a $20 \%$ reduction in breast cancer mortality in women of 74 years and younger. Since women of $75+$ years are often excluded from randomized control studies, observation studies and modeling predictions provide the next best data available for evaluating the risks and benefits of screening mammograms [12]. Although observational studies are subject to potential bias, there is a mortality reduction benefit reported in these observational studies, forcing us to consider whether the benefits from screening mammography are underrepresented. One study investigated the relationship between prior regular mammography use, stage of breast cancer at diagnosis and breast cancer mortality in the elderly and found that older women, who did not get regular mammograms were diagnosed with higher-stage breast cancers than regular users. Furthermore, these individuals also had a greater risk of dying from their breast cancer compared to regular users [16]. In addition to providing a mortality benefit, screening mammograms are also noted to have a higher invasive cancer detection rate in older women secondary to decreasing breast density with age, resulting in higher sensitivity and specificity, decreasing the number of false positive mammogram and biopsy results [12].

The most cited harms of screening mammogram in the elderly include evaluation of coexisting comorbidities, and concern for overdiagnosis and overtreatment of indolent disease $[12,17]$. Overdiagnosis is defined as the detection of breast cancer that would not have been detected in a woman's lifetime in the absence of screening [17]. One study aimed to quantify the benefits and harms of mammography screening after 74 years by focusing on the amount of overdiagnosis of breast cancer by using three microsimulation models that simulated a cohort of women born in 1960 with average life expectancies of 13 years of age at age 74 [18]. The models predicted that there were 7.8 - 11.4 life years gained per 1,000 screening mammograms at age 74 years, 4.8 - 7.8 life years gained per 1,000 screens at age 80 years, and $1.4-2.4$ life years gained per 1,000 screens at 90 years [18]. The benefits of screening mammography outweighed the risks until the age of 90 years and the percentage of screen-detected breast cancers that were overdiagnosed increased with age [18]. There was a large difference in the range of estimates of overdiagnosis reported in this study. With increasing life expectancies, these models likely underestimated the benefits of screening mammography in the elderly, since age was an important factor that was incorporated into the models.

Multiple coexisting comorbidities also make it difficult for clinicians to advocate for screening mammogram in the elderly. Interestingly, some studies suggest that individuals with even mild to moderate comorbidities still benefit from screening mammography by observed decreased relative risk of death in the elderly $[12,19]$. As expected, as the severity of the comorbidities increase, the survival benefits of screening mammography decrease [20]. Although comorbidities were not specifically evaluated in our study, the number of comor- 
bidities should not be used as a reason to avoid screening in the elderly, especially with recent observations of increasing life expectancy. Unfortunately, there is no way to standardize comorbidity assessments and as a direct result, clinicians sometimes overemphasize comorbidities and discourage screening in the elderly. Over the last decade, a growing number of studies have included measures of comorbidities but with little consistency in measurement, resulting in a lack of comparability across research settings [21]. This could be another reason why the elderly in our sample were noted to have lower screening mammogram percentages.

Just as the elderly are underrepresented in clinical trials involving breast cancer prevention, they are also understated in clinical trials involving breast cancer treatment [22]. Much of what is known regarding cancer treatment in the elderly is extrapolated from clinical trials conducted in younger, healthier patients, leading to a systemic difference in treatment, disparities, and health outcomes between the two different populations [23]. Many studies suggest that elderly breast cancer patients undergo a comprehensive geriatric assessment to help physicians objectively evaluate their patients and identify deficits that would not be apparent by history and physical examination alone [24, 25]. Underutilization of tools, especially in underserved communities, may also be reasons for over and undertreatment of breast cancer.

Breast cancers detected with screening mammography are less likely to metastasize to lymph nodes and more likely to be treated with breast conservation treatment without chemotherapy compared to those women who declined screening $[14,26]$. In our study, individuals that underwent screening mammograms were more likely to have stage 0 or early staged breast cancers and estrogen and luminal A hormone expression which are considered favorable characteristics [26]. Interestingly, epidemiology studies have reported that triple-negative breast cancers are more common in women of African ancestry in comparison to others ethnic groups $[27,28]$. Since many of our patients are of African ancestry, we were interested to see if elderly African American patients in our sample had significant representation of triple-negative breast cancers compared to Caucasian patients. In both races and age groups, there were more luminal A type observed; there was no statistical difference reported between races for triple-positive, negative, or luminal A type breast cancers. Studies cite that racial disparities in triple-negative breast cancer are secondary to both biologic (e.g., tumor heterogeneity, population genetics, somatic genomic mutations, gene expression) and non-biologic changes (e.g., poverty, social stressors, lack of healthcare access, obesity) $[28,29]$. Our research question did not focus on individual disparities, but it is likely that these factors influenced the results of our study.

\section{Conclusions}

Decreased compliance to screening mammography is observed in the elderly living in lower economically developed areas. Since the elderly are often underrepresented in clinical trials, organizations do not have sufficient data to advocate for screening mammography in this age group. With increasing life expectancy, however, observational studies have demonstrated a mortality benefit with screening mammography by early breast cancer detection rates, favorable breast cancer characteristics and potentially higher cure rates. In our study, both the $70-75$ age group and 75+ age group had predominantly early stage, luminal A hormone expression breast cancers consistent with the above observational studies, prompting us to support formation of comprehensive and discrete guidelines for the elderly.

When the elderly concurrently live in underserved areas, additional socioeconomic factors can also affect compliance to screening mammography. Individuals of lower socioeconomic status likely have decreased compliance to screening mammography secondary to lack of education and accessibility to proper health care services. Although individual social disparities were not evaluated, it is likely they influenced screening mammography compliance in our study. Future studies can investigate how individual socioeconomic factors can affect screening mammography compliance in the elderly so we can better understand how to promote breast cancer prevention in the elderly residing in underserved communities.

\section{Acknowledgments}

Thanks to the Franciscan Health Olympia Fields Comprehensive Cancer Center.

\section{Financial Disclosure}

We did not receive funding for this project. Franciscan Health Graduate Medical Education, however, will pay for publication cost.

\section{Conflict of Interest}

None to declare.

\section{Informed Consent}

Our institutional review board waived informed consent for our study.

\section{Author Contributions}

Shruti Sharma: conceptualization, methodology, formal analysis, investigation, resources, data curation, writing-original draft, review and editing, visualization. Amy Stein: software, validation, formal analysis, writing-review and editing. Dixita Patel: investigation, resources, data curation, writing-review and editing. Sushma Pavuluri: investigation, resources, data curation, writing-review and editing. Binal Patel: investigation, resources, data curation, writing-review and editing. Nadia Qureshi: investigation, resources, and data curation. 
Imran Hasnuddin: investigation, resources, and data curation. Tsvetelina Todorova: investigation, resources, and data curation. Masood Ghouse: conceptualization, methodology, formal analysis, investigation, writing-review and editing, and supervision. Krishnan Srinivasan: writing-review and editing, supervision.

\section{Data Availability}

The data used to support the findings of this study may be released upon application to the Institutional Review Board titled "Franciscan Health Institutional Review Board EST, Mishawaka, Indiana", who can be contacted at this email: Kathleen. Kioussopoulos@franciscanalliance.org.

\section{References}

1. Sung H, Ferlay J, Siegel RL, Laversanne M, Soerjomataram I, Jemal A, Bray F. Global cancer statistics 2020: GLOBOCAN estimates of incidence and mortality worldwide for 36 cancers in 185 countries. CA Cancer J Clin. 2021;71(3):209-249.

2. Warner E. Clinical practice. Breast-cancer screening. N Engl J Med. 2011;365(11):1025-1032.

3. Mavaddat N, Peock S, Frost D, Ellis S, Platte R, Fineberg E, Evans DG, et al. Cancer risks for BRCA1 and BRCA2 mutation carriers: results from prospective analysis of EMBRACE. J Natl Cancer Inst. 2013;105(11):812-822.

4. Surveillance, Epidemiology, and End Results (SEER) Program (www.seer.cancer.gov) SEER*Explorer: Breast. Recent Trends in Seer Age-Adjusted Incidence Rates, 2000-2018. By Age, Observed SEER Incidence Rate, Female, All Races, All Stages.

5. Kotwal AA, Schonberg MA. Cancer screening in the elderly: a review of breast, colorectal, lung, and prostate cancer screening. Cancer J. 2017;23(4):246-253.

6. National Cancer Database (NCDB). NCDB Public Benchmark Reports. Cases diagnosed between 20092018. breast. Case types: class of case 00 and class of case 10-14. Variable: age. hospital: comprehensive community cancer program. Geography: All States.

7. National Cancer Database (NCDB): NCDB private benchmark reports. Cases diagnosed between 2009-2018. Breast. Case types: class of case 00 and class of case 10 14. Variable: age: hospital: comparison of our facility vs all comprehensive community cancer programs. Geography: All States.

8. Surveillance, Epidemiology and End Results (SEER) Program (www.seer.cancer.gov). Annual frequency of diagnosis- SEER diagnostic groups -10 year review of our facility of all malignancies. Report \#1-03-01sv_4.

9. Medina LD, Shannon S, Jonathan V. Living longer: historical and projected life expectancy in the United States, 1960 to 2060. Current population reports, U.S. Census Bureau. 2020.

10. Robert SA, Strombom I, Trentham-Dietz A, Hampton JM, McElroy JA, Newcomb PA, Remington PL. Socio- economic risk factors for breast cancer: distinguishing individual- and community-level effects. Epidemiology. 2004;15(4):442-450.

11. American Cancer Society. Breast Cancer Facts \& Figures 2019-2020. Atlanta: American Cancer Society, Inc. 2019.

12. Lee CS, Moy L, Joe BN, Sickles EA, Niell BL. Screening for breast cancer in women age 75 years and older. AJR Am J Roentgenol. 2018;210(2):256-263.

13. National Center for Health Statistics. Health, United States, 2019: Table \#033 Hyattsville, MD. 2021. https:// www.cdc.gov/nchs/hus/contents2019.htm.

14. Smith ML, Hochhalter AK, Ahn S, Wernicke MM, Ory MG. Utilization of screening mammography among middle-aged and older women. J Womens Health (Larchmt). 2011;20(11):1619-1626.

15. Diab SG, Elledge RM, Clark GM. Tumor characteristics and clinical outcome of elderly women with breast cancer. J Natl Cancer Inst. 2000;92(7):550-556.

16. McCarthy EP, Burns RB, Freund KM, Ash AS, Shwartz M, Marwill SL, Moskowitz MA. Mammography use, breast cancer stage at diagnosis, and survival among older women. J Am Geriatr Soc. 2000;48(10):1226-1233.

17. Braithwaite D, Demb J, Henderson LM. Optimal breast cancer screening strategies for older women: current perspectives. Clin Interv Aging. 2016;11:111-125.

18. van Ravesteyn NT, Stout NK, Schechter CB, Heijnsdijk EA, Alagoz O, Trentham-Dietz A, Mandelblatt JS, et al. Benefits and harms of mammography screening after age 74 years: model estimates of overdiagnosis. J Natl Cancer Inst. 2015;107(7):djv103.

19. McPherson CP, Swenson KK, Lee MW. The effects of mammographic detection and comorbidity on the survival of older women with breast cancer. J Am Geriatr Soc. 2002;50(6):1061-1068.

20. Satariano WA, Ragland DR. The effect of comorbidity on 3-year survival of women with primary breast cancer. Ann Intern Med. 1994;120(2):104-110.

21. Williams GR, Deal AM, Lund JL, Chang Y, Muss HB, Pergolotti M, Guerard EJ, et al. Patient-reported comorbidity and survival in older adults with cancer. Oncologist. 2018;23(4):433-439.

22. Sedrak MS, Freedman RA, Cohen HJ, Muss HB, Jatoi A, Klepin HD, Wildes TM, et al. Older adult participation in cancer clinical trials: A systematic review of barriers and interventions. CA Cancer J Clin. 2021;71(1):78-92.

23. Hurria A, Levit LA, Dale W, Mohile SG, Muss HB, Fehrenbacher L, Magnuson A, et al. Improving the evidence base for treating older adults with cancer: American society of clinical oncology statement. J Clin Oncol. 2015;33(32):3826-3833.

24. Balducci L, Extermann M, Carreca I. Management of breast cancer in the older woman. Cancer Control. 2001;8(5):431-441.

25. Fusco D, Allocca E, Rocco Villani E, Franza L, Laudisio A, Colloca G. An update in breast cancer management for elderly patients. Transl Cancer Res. 2018;7:S319-28.

26. Al-Zawi ASA, Adamczyk B, Wejman-Matela A, Sathananthan S. Histopathological types of operable early breast cancer in the elderly: is there a special pat- 
tern? A retrospective, multicentre study. Med Res J. 2018;3(1):10-14.

27. Siddharth S, Sharma D. Racial Disparity and Triple-Negative Breast Cancer in African-American Women: A Multifaceted Affair between Obesity, Biology, and Socioeconomic Determinants. Cancers (Basel). 2018;10(12):514.

28. Danforth DN, Jr. Disparities in breast cancer outcomes be- tween Caucasian and African American women: a model for describing the relationship of biological and nonbiological factors. Breast Cancer Res. 2013;15(3):208.

29. Prakash O, Hossain F, Danos D, Lassak A, Scribner R, Miele L. Racial disparities in triple negative breast cancer: a review of the role of biologic and non-biologic factors. Front Public Health. 2020;8:576964. 